\title{
Quantum oscillations and high carrier mobility in the delafossite $\mathrm{PdCoO}_{2}$
}

\author{
Clifford W. Hicks, ${ }^{1}$ Alexandra S. Gibbs, ${ }^{1,2}$ Andrew P. Mackenzie,,${ }^{1, *}$ \\ Hiroshi Takatsu, ${ }^{3}$ Yoshiteru Maeno, ${ }^{4}$ and Edward A. Yelland ${ }^{1,5}$ \\ ${ }^{1}$ Scottish Universities Physics Alliance (SUPA), School of Physics and Astronomy, \\ University of St. Andrews, St. Andrews KY16 9SS, United Kingdom \\ ${ }^{2}$ School of Chemistry and EaStCHEM, University of St. Andrews, \\ North Haugh, St. Andrews KY16 9ST, United Kingdom \\ ${ }^{3}$ Department of Physics, Tokyo Metropolitan University, Tokyo 192-0397, Japan \\ ${ }^{4}$ Department of Physics, Graduate School of Science, Kyoto University, Kyoto 606-8502, Japan \\ 5 SUPA, School of Physics and Astronomy, and Centre for Science at Extreme Conditions, \\ University of Edinburgh, Mayfield Road, Edinburgh EH9 3JZ, United Kingdom
}

(Dated: 23 July 2012)

\begin{abstract}
We present de Haas-van Alphen and resistivity data on single crystals of the delafossite $\mathrm{PdCoO}_{2}$. At $295 \mathrm{~K}$ we measure an in-plane resistivity of $2.6 \mu \Omega-\mathrm{cm}$, making $\mathrm{PdCoO}_{2}$ the most conductive oxide known. The low-temperature in-plane resistivity has an activated rather than the usual $T^{5}$ temperature dependence, suggesting a gapping of effective scattering that is consistent with phonon drag. Below $10 \mathrm{~K}$, the transport mean free path is $\sim 20 \mu \mathrm{m}$, approximately $10^{5}$ lattice spacings and an astoundingly high value for flux-grown crystals. We discuss the origin of these properties in light of our data.
\end{abstract}

PACS numbers:

A number of discoveries, for example graphene and the superconductivity in the sodium cobaltate system, have focused attention on the properties of two-dimensional conduction on triangular lattices. One of the most prominent families of such materials is the delafossites $\mathrm{ABO}_{2}$, where the $\mathrm{A}$ atoms $(\mathrm{Pt}, \mathrm{Pd}, \mathrm{Ag}$ or $\mathrm{Cu}$ ) are directly bonded to each other in triangular-lattice sheets, separated by $\mathrm{BO}_{2}$ layers. Possibilities for $\mathrm{B}$ include $\mathrm{Cr}, \mathrm{Co}$, $\mathrm{Fe}, \mathrm{Al}$ and $\mathrm{Ni}$ [1, 2]. The delafossite family includes band insulators and semiconductors [2, 3], transparent semiconductors 4], metallic magnets [5], candidate thermoelectrics [6], and magnetic and magneto-electric insulators [7. There are also nonmagnetic metals that present a combination of strong anisotropy, high carrier density and high mobility that is unique for bulk materials [3, 8. In the long term, the delafossites may be a candidate family for multilayer technologies benefiting from their broad range of physical properties. High purity is a prerequisite for such developments, and so the extremely high conductivity of nonmagnetic delafossite metals is worthy of investigation. Here, we concentrate on the most extensively studied material, $\mathrm{PdCoO}_{2}$.

$\mathrm{PdCoO}_{2}$ crystallizes in the space group $R \overline{3} m\left(D_{3 d}^{5}\right)$. The lattice parameters of the hexagonal unit cell at $298 \mathrm{~K}$ are $a=b=2.830 \AA$, and $c=17.743 \AA$ 9. Single crystals have been grown by several groups 9 11. The Co atoms have formal valence +3 and configuration $3 d^{6}$, and calculations place the Fermi level $E_{F}$ between filled pseudo- $t_{2 g}$ and empty pseudo- $e_{g}$ levels [12 14. The Pd sheets therefore dominate the conductivity, leading to high transport anisotropy. The $\mathrm{Pd}$ atoms have formal valence +1 , in configuration $4 d^{9-x} 5 s^{x}$. A key issue in understanding the physics of $\mathrm{PdCoO}_{2}$ is the relative importance of the $4 d$ and $5 s$ states to the conduction. We will return to this in light of our data.

$\mathrm{PdCoO}_{2}$ is nonmagnetic, and so shows none of the frustration effects commonly associated with magnetism in triangular lattices. Band structure calculations [12, 15] and angle-resolved photoemission spectroscopy (ARPES) data [10] indicate that it has a broad, half-filled conduction band, leading to a single, nearly two-dimensional Fermi surface (FS) with a rounded hexagonal cross section. This is different from graphene or graphite, since the $\mathrm{Pd}$ atoms form a simple triangular array rather than a honeycomb lattice.

At room temperature, $\mathrm{PdCoO}_{2}$ is the most conductive oxide known. To measure the in-plane resistivity $\rho_{a b}$, we cut three needle-like samples from a $6 \mu \mathrm{m}$-thick single crystal, of length $\sim 400 \mu \mathrm{m}$ and widths 39,42 and $120 \mu \mathrm{m}$. The measured resistances of all samples were consistent with $\rho_{a b}(295 \mathrm{~K})=2.6(2) \mu \Omega$-cm: two and four times, respectively, less resistive than other oxides known for their high conductivity, $\mathrm{SrMoO}_{3}$ and $\mathrm{ReO}_{3}$ [16, 17. Among the elemental metals, only $\mathrm{Cu}, \mathrm{Ag}$ and $\mathrm{Au}$ have lower resistivities $(1.7,1.6$ and $2.2 \mu \Omega$-cm, respectively). The high conductivity of $\mathrm{PdCoO}_{2}$ is especially surprising considering that the $\mathrm{CoO}_{2}$ layers do not contribute itinerant carriers: the carrier density is $30 \%$ that of $\mathrm{Cu}$.

To study the origin of this high conductivity, we combined a de Haas-van Alphen (dHvA) study, using a piezoresistive torque method [18, with high-resolution resistivity measurements. The crystals were grown as described in Ref. 9]. They are clean: the resistivity samples have $\rho_{a b}(295 \mathrm{~K}) / \rho_{a b}(0) \approx 250$. Torque measurements were carried out between $50 \mathrm{mK}$ and $20 \mathrm{~K}$, on a crystal $\approx 120 \mu \mathrm{m}$ across and $30 \mu \mathrm{m}$ thick; example torque data are shown in Fig. 1. That the measurement current was not heating the samples was checked with simulta- 

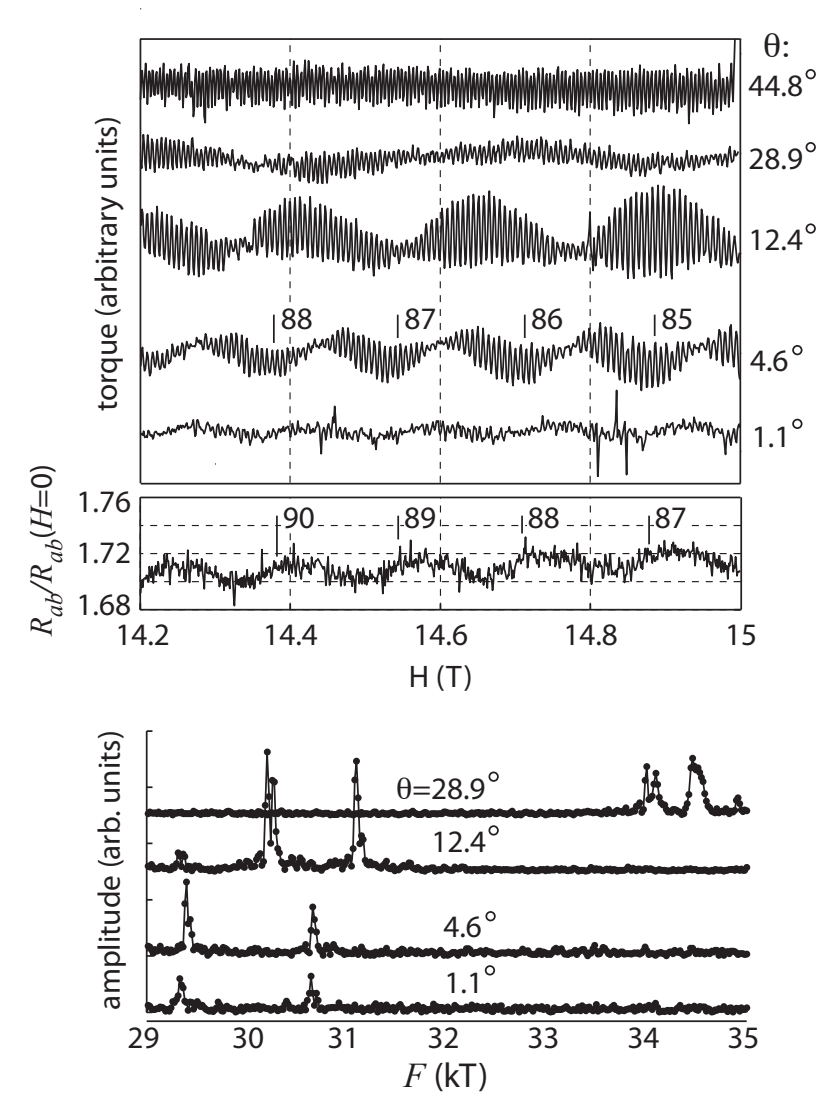

FIG. 1: Upper panel: torque curves against $H$, at the indicated angles $\theta$ between $\mathbf{H}$ and the $c$-axis, and magnetoresistance of a resistivity sample. The ticks on this curve and the $4.6^{\circ}$ torque curve label the cycle number of the difference frequency: the troughs in the resistance oscillations match up with points where the torque oscillation amplitude (and likely magnetic interaction) is largest. Lower panel: Fourier transforms over the field range 8.1-15 $\mathrm{T}$.

neous measurement of dHvA oscillations in $\mathrm{Sr}_{2} \mathrm{RuO}_{4}$, a renormalized Fermi liquid with well-known quasiparticle masses. Using a rotatable sample stage, the field $\mathbf{H}$ could be rotated by an angle $\theta$ from the crystal $c$-axis towards the $F$ symmetry point of the first Brillouin zone (as illustrated in Fig. 22). The temperature dependence of the dHvA effect was studied at $\theta=-6^{\circ}$, and the $\theta$ dependence at $T=0.7 \mathrm{~K}$.

The data in Fig. 1 are consistent with $\mathrm{PdCoO}_{2}$ being a high purity, high carrier concentration, quasi-2D metal. At each angle $\theta$ we observe two large dHvA frequencies that differ by only $\sim 3 \%$, and that vary approximately as $1 / \cos (\theta)$, confirming the overall cylindrical form of the FS. We also observe significant magnetic interaction (MI), an effect seen in the highest purity metals [19. Most torque curves show a common characteristic of MI, a strong difference frequency amplitude, visible in Fig. 1 as an oscillation matching the beating of the two high frequencies. The difference frequency also appears in the magnetoresistance, ruling out torque interaction as its origin.

Considerable insight into the physics of $\mathrm{PdCoO}_{2}$ can be obtained from detailed analysis of the dHvA data. Following 20, and 21, the corrugations on nearlycylindrical Fermi surfaces can be expanded into harmonic components with amplitudes $k_{\mu \nu}$ :

$$
k_{F}\left(\phi, k_{z}\right)=\sum_{\mu, \nu} k_{\mu \nu} \cos \mu \phi\left\{\begin{array}{l}
\cos \nu d k_{z} \\
\sin \nu d k_{z}
\end{array},\right.
$$

where $k_{z}$ is the $z$ component of the wavevector, $d=c / 3$ the interlayer spacing, and $\phi$ the azimuthal angle. $k_{00}$ is the average radius of the FS, and the remaining $k_{\mu \nu}$ are the amplitudes of harmonic components of the corrugation. The components considered in this paper are illustrated in Fig. 2 The triangular lattice of the Pd sheets allows components with $\mu=0,6,12, \ldots$ with an even (i.e. cosine) dependence on $k_{z}$. In addition, because $\mathrm{PdCoO}_{2}$ lacks mirror symmetry along the $c$-axis, $\mu=3,9,15, \ldots$ are allowed with odd (i.e. sine) $k_{z}$ dependence.

Since $k_{z}$-independent warpings cannot be obtained from dHvA frequencies, we set $k_{60}=0.040(3)$ and $k_{12,0}=0.007(2) \AA^{-1}$ to match the ARPES Fermi surface [10. With these amplitudes fixed, we then allow $k_{00}, k_{01}, k_{02}$ and $k_{31}$ to vary to obtain the best match to the dHvA frequencies, setting all higher-order amplitudes to zero. The FS cross-sectional areas in the fit to the data were calculated by numerical integration 22 . The fit and the resulting FS are shown in Fig. 2 The data constrain the relative signs of $k_{01}, k_{02}$ and $k_{31} ; k_{01}$ is taken to be $>0$ to match electronic structure calculations.

Numerical integration of the volume enclosed by the FS yields a carrier density of 1.005(1) per formula unit, based on room-temperature values for $a$ and $b$. If $a$ and $b$ shrink by $\sim 0.25 \%$ at low temperatures then the carrier density becomes almost exactly one: the FS of $\mathrm{PdCoO}_{2}$ arises, to high precision, from a single half-filled band.

As mentioned above, a key issue for understanding $\mathrm{PdCoO}_{2}$ is the relative importance of the $\mathrm{Pd} 4 d$ and $5 s$ orbitals in states near the Fermi level. The dHvA masses $\left[m^{*} \equiv\left(\hbar^{2} / 2 \pi\right) d A / d E\right.$, where $A$ is the FS cross-sectional area] provide the first piece of evidence that the $5 s$ component is more important than has usually been assumed. Our data yield $m^{*}=1.45(5) m_{e}$ and $1.53(3) m_{e}$ for the 29.3 and $30.7 \mathrm{kT}$ orbits, respectively $\left(\right.$ at $\left.\theta=-6^{\circ}\right)$ [23], corresponding to an electronic specific heat of approximately $1 \mathrm{~mJ} / \mathrm{mol}-\mathrm{K}^{2}$, in fairly good agreement with a measured value of $1.28 \mathrm{~mJ} / \mathrm{mol}^{-\mathrm{K}^{2}}$ [9, 23]. These masses would be very low for a $d$-orbital-derived FS. For comparison, Pd metal (which has atomic spacing $2.75 \AA$, just $3 \%$ less than in $\left.\mathrm{PdCoO}_{2}\right)$ has a mainly $5 s$-derived electronlike Fermi pocket, compensated by a $4 d$-derived open hole surface, and the dHvA masses of these surfaces are $\sim 2.0$ and $5-10 m_{e}$, respectively [24, 25. The $\mathrm{PdCoO}_{2} \mathrm{dHvA}$ 

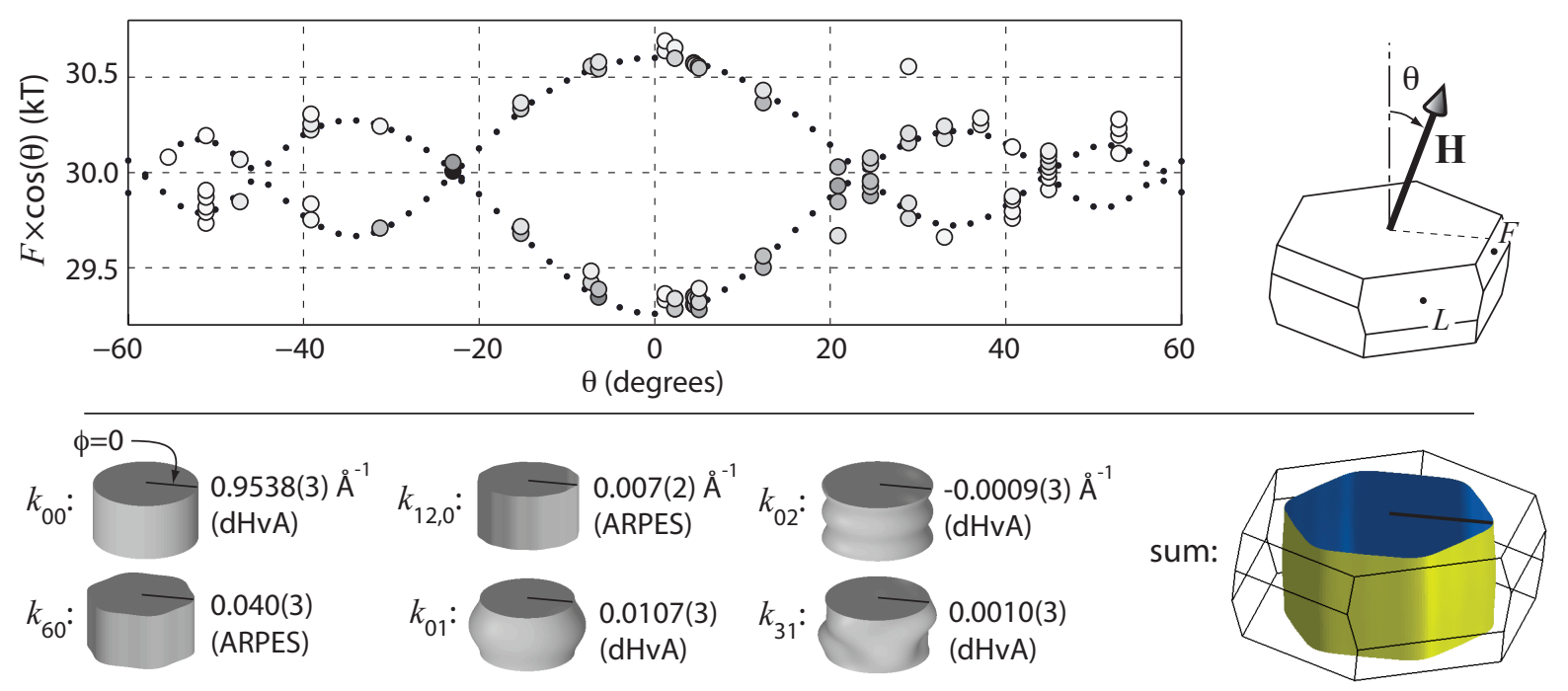

FIG. 2: Top: Observed dHvA frequencies $F$, obtained by Fourier transform over the field range 8.1 to 15 T (circles; darker shading indicates larger amplitude), and a fit (small points). Bottom: the separate harmonic components included in the fit, and their amplitudes. $k_{60}$ and $k_{12,0}$ are obtained from ARPES data [10], and $k_{00}, k_{01}, k_{02}$ and $k_{31}$ from the fit. $k_{01}$ gives the overall Yamaji-type form to $F(\theta)$ and $k_{02}$ an asymmetry between the upper and lower frequencies. $k_{31}$ gives an asymmetry between $\theta>0$ and $\theta<0$, which is allowed by the lack of mirror symmetry in the crystal structure. The Fermi surface that results from summing these components is shown at right.

masses are much closer to that of the $\mathrm{Pd} 5 s$ than the $\mathrm{Pd}$ $4 d$ surface.

The form of the FS warping also emphasizes the role played by the $5 s$ component. The dominant warping is $k_{01}$, suggesting interlayer transport by direct $\mathrm{Pd}-\mathrm{Pd}$ overlap, which would rely on the extended $5 s$ component to cross the $6 \AA$ interlayer separation. Hopping via the Co atoms would allow transport through the more compact $d$ orbitals, but connects interlayer next-nearest-neighbor $\mathrm{Pd}$ sites (through the path $\mathrm{Pd}-\mathrm{O}-\mathrm{Co}-\mathrm{O}-\mathrm{Pd}$, illustrated in Fig. 5b of [15]), leading to a large $k_{31}$. Published calculations indeed show bumps on the FS with significant Co character that give a large $k_{31}[12,15]$. The dHvA data, however, show that $k_{01}$ is far larger than $k_{31}$.

Hybridization between the $\mathrm{Pd} 4 d_{z^{2}}$ and $5 s$ orbitals has been discussed in [3, 13, 26]. Reports on electronic structure calculations have tended to mention the likelihood of a $5 s$ component at $E_{F}$ but emphasize the $4 d$ contribution [12 15]. However in a calculation of $\mathrm{PdCoO}_{2}$ without the $\mathrm{CoO}_{2}$ spacer layers, i.e. bare Pd sheets, a $5 \mathrm{~s}$ manifold that straddles $E_{F}$ is clearly visible (Fig. 9 of Ref. [13]), and remains apparent in full $\mathrm{PdCoO}_{2}$ band structures.

To analyze our data and compare with previous calculation, we performed electronic structure calculations on $\mathrm{PdCoO}_{2}$ using the general potential linearized augmented plane wave method as implemented in the WIEN2K package. We calculated $20,000 k$ points in the full Brillouin zone, and studied the effects of an on-site repulsion $U_{\text {eff }}$ at the Co atoms. For $U_{\text {eff }}=0$, we re- cover previously reported results, and find $\mathrm{dHvA}$ masses of around $1.9 m_{e}$, larger than the measured value. Introducing a modest $U_{\text {eff }}$ reduces the calculated masses towards the experimental values and simultaneously gives a ratio between $k_{31}$ and $k_{01}$ that is in better agreement with experiment (Table 1). The high sensitivity of the in-plane masses, which arise from Pd-Pd bonding, to the Co site Coulomb repulsion appears surprising. A possible interpretation is that the $\mathrm{Pd} 5 s$ component is extended enough to be sensitive to the charge distribtuion in the $\mathrm{CoO}_{2}$ layers; this should be the subject of further investigation.

Although conduction by $5 s$ states might account for the scale of the conductivity of $\mathrm{PdCoO}_{2}$ at room temperature, the low temperature resistivity has additional intriguing features. Measurement is challenging because of the tiny resistance of typical samples. By using transformers mounted at $1 \mathrm{~K}$ to provide low-noise $(150 \mathrm{pV} / \sqrt{\mathrm{Hz}})$ passive amplification, we obtained the data shown in Fig. 3 . The difference between $\rho_{c}$ and $\rho_{a b}$

TABLE I: LDA $+\mathrm{U}$ results, and experimental data. $U_{\text {eff }}$ is the effective on-site Coulomb repulsion at the Co sites. $m_{\Gamma}^{*}$ is the dHvA mass for the orbit in the $k_{z}=0$ plane, and $m_{Z}^{*}$ the $k_{z}= \pm \pi$ plane.

\begin{tabular}{llllll}
\hline \hline & $m_{\Gamma}^{*}$ & $m_{Z}^{*}$ & $k_{01}\left(\AA^{-1}\right)$ & $k_{31}\left(\AA^{-1}\right)$ & $k_{31} / k_{01}$ \\
\hline$U_{\text {eff }}=0 \mathrm{eV}$ & $1.90 m_{e}$ & $1.82 m_{e}$ & 0.0039 & 0.0172 & 4.4 \\
$U_{\text {eff }}=5 \mathrm{eV}$ & 1.66 & 1.52 & 0.0181 & 0.0029 & 0.2 \\
experiment & 1.53 & 1.45 & $0.0107(3)$ & $0.0010(3)$ & 0.1
\end{tabular}



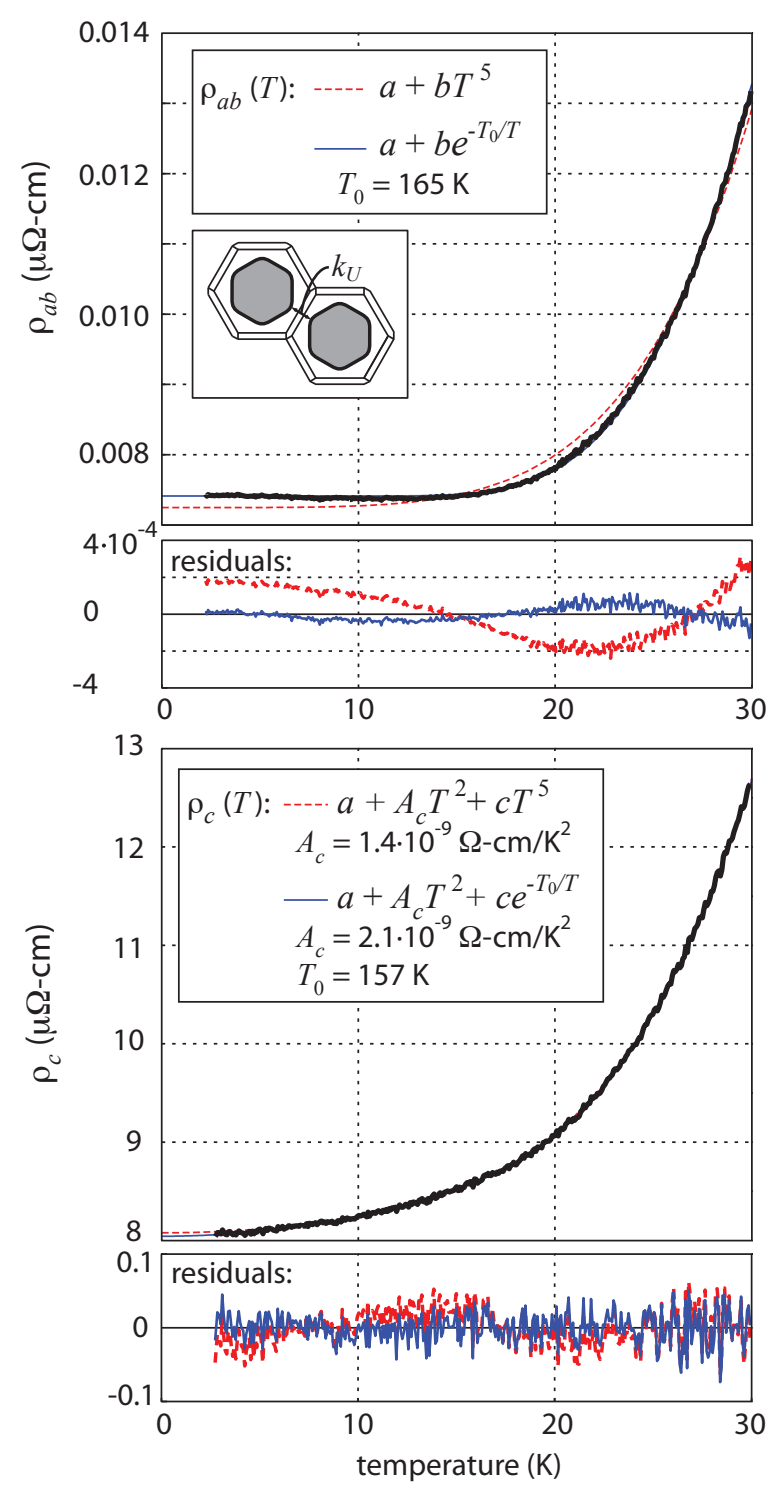

FIG. 3: In-plane $\mathrm{PdCoO}_{2}$ resistivity $\rho_{a b}(T)$ (top), and $c$-axis resistivity $\rho_{c}(T)$ (bottom), with fits. At low $T, \rho_{c}$ has a $T^{2}$ dependence, while $\rho_{a b}$ is nearly temperature-independent. The inset in the $\rho_{a b}$ panel illustrates $k_{U}$, the minimum wavenumber for Umklapp scattering.

is striking: while $\rho_{c}$ shows a $T^{2}$ dependence at low temperatures, $\rho_{a b}$ becomes almost temperature-independent.

Below $30 \mathrm{~K}, \rho_{a b}(T)$ is much better described by an activated form than the usual electron-phonon $T^{5}$ form (Fig. 3). This may be a consequence of phonon drag, in which the phonon population is pushed out of equilibrium by charge carrier flow. It is a well-known phenomenon in low-temperature thermopower, but has been seen in resistivity only in the alkali metals 27 . In clean materials momentum is transferred to the lattice through Umklapp processes. For the alkali metals and for in-plane conduction in $\mathrm{PdCoO}_{2}$, the Fermi surface is closed, resulting in an activation energy $k_{B} T_{U}=\hbar c k_{U}$, where $k_{U}$ is the minimum wavenumber for Umklapp processes (illustrated in Fig. 3) and $c$ is the speed of sound. For $T \ll T_{U}$, electron-phonon scattering transfers momentum between electrons and phonons but does not relax the momentum of the combined system. $k_{U} \approx 0.5 \AA^{-1}$ in $\mathrm{PdCoO}_{2}$. Fits to $\rho_{a b}(T)$ for two samples yield activation temperatures of 165 (Fig. 3) and $168 \mathrm{~K}$ (not shown), giving $c \approx 4400 \mathrm{~m} / \mathrm{s}$. This is in fair agreement with specific heat measurements, where the magnitude of the $T^{3}$ term, $0.0619 \mathrm{~mJ} / \mathrm{mol}^{-\mathrm{K}^{4}}$ [9], yields, for isotropic sound propagation, $c=3650 \mathrm{~m} / \mathrm{s}$. This analysis works equally well for potassium [28.

Fermi liquids usually have a $T^{2}$ low-temperature resistivity due to electron-electron scattering. $A_{c}$ in $\rho_{c}(T)=$ $\rho_{c}(0)+A_{c} T^{2}$ is $2 \cdot 10^{-9} \Omega-\mathrm{cm} / \mathrm{K}^{2} . \rho_{a b}(T)$ shows a very small resistivity upturn at low $T$, and the upper limit we can place on $A_{a b}$ is $1.0 \cdot 10^{-12} \Omega-\mathrm{cm} / \mathrm{K}^{2}$. This is not a particularly small value for metals with electronic specific heats comparable to $\mathrm{PdCoO}_{2}$ [29], but it is significantly smaller than $A_{c}$ scaled by the low- $T$ resistive anisotropy $\left(\rho_{c}(0) / \rho_{a b}(0)=1000\right)$. In the alkali metals $\mathrm{K}$ and $\mathrm{Na}$ the closed FS geometry is known to partially suppress electron-electron Umklapp processes, reducing $A$ by about a factor of ten [30. Such a suppression is also likely to apply to $A_{a b}$ of $\mathrm{PdCoO}_{2}$.

The combination of resistivity and $\mathrm{dHvA}$ data also shows that transport electron-phonon coupling in $\mathrm{PdCoO}_{2}$ is very small over an extended temperature range. In Ref. [9], $\rho_{a b}(T)$ of $\mathrm{PdCoO}_{2}$ for $0<T<500 \mathrm{~K}$ is fitted to a sum of the Bloch-Gruneisen form and an Einstein mode contribution. These fits return Debye and Einstein temperatures $\Theta_{D}$ and $\Theta_{E}$, and transport electron-phonon couplings scaled by the the plasma frequency squared, $\lambda_{D, \mathrm{tr}} / \Omega_{p}^{2}$ and $\lambda_{E, \mathrm{tr}} / \Omega_{p}^{2}$ 31. The dHvA data here allow determination of the plasma frequency: $\Omega_{p, x x}^{2}=n e^{2} / m \epsilon_{0}=\left(7.2(1) \cdot 10^{15} \mathrm{sec}^{-1}\right)^{2}$. Performing the same fit over the range $60<T<300 \mathrm{~K}$, where the lower bound excludes the region strongly affected by phonon drag, we obtain $\Theta_{D}=329 \mathrm{~K}$ and $\Theta_{E}=1106 \mathrm{~K}$, in good agreement with [9]. $\lambda_{D, \text { tr }}$ and $\lambda_{E, \text { tr }}$ come to 0.043 and 0.023 , respectively, which are exceptionally low values: $\mathrm{Cu}$ has $\lambda_{D \text {,tr }}=0.13$ and $\mathrm{Ca}$, a metal with a particularly low dHvA mass of $\sim 0.6 m_{e}, 0.05$ [32]. These low couplings may also be due to the FS topology, through a partial suppression of Umklapp processes at temperatures $T \sim T_{U}$. Careful calculation would be required to test this hypothesis.

Our measured residual resistivities $\rho_{a b}(T \rightarrow 0)$, between 0.007 and $0.008 \mu \Omega$-cm for two samples, are very low, corresponding to a transport mean free path of $20 \mu \mathrm{m}$, or, in a standard interpretation, a defect density in the $\mathrm{Pd}$ layers of one in $10^{5} 33$. This is a remarkable value for flux-grown crystals. To set it in context, the fractional quantum Hall effect has recently been ob- 
served in $\mathrm{ZnO}$-based heterostructures, where, after years of research, the transport mean free path has been increased to $\sim 1.5 \mu \mathrm{m}$ 34. This is considerably less than what we report here for a naturally two-dimensional material, motivating the study of delafossites as a natural material for multilayer oxide technologies.

In summary, we have observed exceptionally high conductivity in $\mathrm{PdCoO}_{2}$ and mapped its Fermi surface in detail using the de Haas-van Alphen effect. Our data suggest that the conductivity of $\mathrm{PdCoO}_{2}$ is due primarily to $\mathrm{Pd} 5 s$ overlap. For in-plane transport, the contributions to resistivity from electron-phonon, electronelectron and electron-impurity scattering are all anomalously low. The effect of the closed Fermi surface topology on Umklapp processes seems to play a role and is worthy of investigation. It is also possible, however, that scattering is suppressed by some non-standard mechanism not considered in the analysis we have presented. Our results provide motivation for further work on this fascinating material.

We acknowledge useful discussions with J.W. Allen, C. Varma, C.A. Hooley, D.J. Singh, and S. Yonezawa, and practical assistance from P.A. Evans. This work has made use of the resources provided by the Edinburgh Compute and Data Facility (ECDF), which is partially supported by the eDIKT initiative. We acknowledge funding from the UK EPSRC, the MEXT KAKENHI (No. 21340100), the Royal Society and the Wolfson Foundation.

* Electronic address: apm9@st-andrews.ac.uk

[1] M.A. Marquardt, N.A. Ashmore, and D.P. Cann, Thin Solid Films 496, 146 (2006).

[2] R.D. Shannon, D.B. Rogers, and C.T. Prewitt, Inorg. Chem. 10, 713 (1971); C.T. Prewitt, R.D. Shannon, and D.B. Rogers, Inorg. Chem. 10,719 (1971).

[3] D.B. Rogers, R.D. Shannon, C.T. Prewitt, and J.L. Gillson, Inorg. Chem. 10, 723 (1971).

[4] H. Yanagi, T. Hase, S. Ibuki, K. Ueda, and H. Hosono, Appl. Phys. Lett. 78, 1583 (2001), and references therein.

[5] H. Takatsu, S. Yonezawa, S. Fujimoto, and Y. Maeno, Phys. Rev. Lett. 105, 137201 (2010).

[6] D.J. Singh, Phys. Rev. B 76, 085110 (2007).

[7] Fa Wang, and A. Vishwanath, Phys. Rev. Lett. 100, 077201 (2008).

[8] P.F. Carcia, R.D. Shannon, P.E. Bierstedt, and R.B. Flippen, J. Electrochem. Soc. 127, 1974 (1980).

[9] H. Takatsu, S. Yonezawa, S. Mouri, S. Nakatsuji, K. Tanaka, and Y. Maeno, J. Phys. Soc. Japan 76, 104701 (2007).

[10] H.-J. Noh, J. Jeong, J. Jeong, E.-J. Cho, S.B. Kim, Kyoo Kim, B.I. Min, and H.-D. Kim, Phys. Rev. Lett. 102, 256404 (2009).

[11] M. Tanaka, M. Hasegawa, and H. Takei, J. Phys. Soc. Japan 65, 3973 (1996).

[12] V. Eyert, R. Frésard, and A. Maignan, Chem. Mater. 20,
2370 (2008).

[13] R. Seshadri, C. Felser, K. Thieme, and W. Tremel, Chem. Mater. 10, 2189 (1998).

[14] Kyoo Kim, H.C. Choi, and B.I. Min, Phys. Rev. B 80, 035116 (2009).

[15] K.P. Ong, Jia Zhang, J.S. Tse, and Ping Wu, Phys. Rev. $B$ 81, 115120 (2010).

[16] I. Nagai, N. Shirakawa, S.-I. Ikeda, R. Iwasaki, H. Nishimura and M. Kosaka, Appl. Phys. Lett. 87, 024105 (2005).

[17] T.P. Pearsall and C.A. Lee, Phys. Rev. B 10, 2190 (1974).

[18] J.R. Cooper, A. Carrington, P.J. Meeson, E.A. Yelland, N.E. Hussey, L. Balicas, S. Tajima, S. Lee, S.M. Kazakov, and J. Karpinski, Physica C 385, 75 (2003).

[19] D. Shoenberg, Magnetic Oscillations in Metals (Cambridge University Press, Cambridge, England, 1983).

[20] C. Bergemann, A.P. Mackenzie, S.R. Julian, D. Forsythe, and E. Ohmichi, Adv. Phys. 52, 639 (2003).

[21] P.D. Grigoriev, Phys. Rev. B 81, 205122 (2010).

[22] Analytic expressions accurate to first order in $k_{\mu \nu}$ are provided in 20], and more accurate expressions in [21.

[23] See Supplemental Material for discussion of the mass determination, resistive anisotropy, a tight-binding calculation and Dingle analysis.

[24] F.M. Mueller, A.J. Freeman, J.O. Dimmock, and A.M. Furdyna, Phys. Rev. B 1, 4617 (1970).

[25] D.H. Dye, S.A. Campbell, G.W. Crabtree, J.B. Ketterson, N.B. Sandesara, and J.J. Vuillemin, Phys. Rev. B 23, 462 (1981).

[26] M. Tanaka, M. Hasegawa, T. Higuchi, T. Tsukamoto, Y. Tezuka, Shik Shin, and H. Takei, Physica B 245, 157 (1998).

[27] J. Bass, W.P. Pratt, and P.A. Schroeder, Rev. Mod. Phys. 62, 645 (1990).

[28] The $T^{3}$ term of the specific heat of potassium is $2.57 \mathrm{~mJ} / \mathrm{mol}^{4}$ [35], yielding $c=1290 \mathrm{~m} / \mathrm{s} . k_{U}=$ $0.24 \AA^{-1}$, so $\hbar c k_{U} / k_{B}=24 \mathrm{~K}$, in good agreement with the activation temperature obtained from fitting $\rho(T)$ : $23 \mathrm{~K}$ 36.

[29] Aluminum, for example, has $\gamma=1.35 \mathrm{~mJ} / \mathrm{mol}^{-\mathrm{K}^{2}}[\mathrm{E} . \mathrm{H}$. Buyco and F.E. Davis, J. Chem. Eng. Data 15, 518 (1970)], similar to $\mathrm{PdCoO}_{2}$, and $A=2.8 \cdot 10^{-13} \Omega$ $\mathrm{cm} / \mathrm{K}^{2}$ 30].

[30] M. Kaveh, and N. Wiser, Adv. Phys. 33, 257 (1984).

[31] P.B. Allen, and W.W. Schulz, Phys. Rev. B 47, 14434 (1993).

[32] P.B. Allen, Phys. Rev. B 36, 2920 (1987).

[33] A standard Dingle analysis of the dHvA oscillations yields a much lower mean free path of $0.6 \mu \mathrm{m}[23$. However this result should be treated with caution, because as conductivity becomes very high phase smearing due to internal field inhomogeneity becomes more problematic. Observing a Dingle mean free path matching the tranport mean free path of $20 \mu \mathrm{m}$ would require a field homogeneity of one in $10^{5}$, probably difficult to achieve in a material with known Co-based, magnetic impurity phases 9 .

[34] A. Tsukazaki, S. Akasaka, K. Nakahara, Y. Ohno, H. Ohno, D. Maryenko, A. Ohtomo, and M. Kawasaki, $\mathrm{Na}$ ture Materials 9, 889 (2010).

[35] W.H. Lien and N.E. Phillips, Phys. Rev. 133, A1370 (1964).

[36] D. Gugan, Proc. R. Soc. Lond. A 325, 223 (1971). 


\section{SUPPLEMENTAL MATERIAL}

Mass determination. The Lifshitz-Kosevich fits used to determine the cyclotron masses at $\theta=-6^{\circ}$ are shown in Fig. 4. The difference frequency probably appears as a result of magnetic interaction. If data below $1.3 \mathrm{~K}$ are excluded from the 29.3 and $30.7 \mathrm{kT}$ fits, out of concern that magnetic interaction might affect the observed amplitudes, then masses of 1.50 and $1.66 m_{e}$ are obtained, respectively.

Specific heat. The electronic specific heat is given by $\gamma \equiv C_{e l} / T=\pi k_{B}^{2} m^{*} / 3 d \hbar^{2} \times V$, where $V$ is the volume per mole. If $m^{*}$ is taken to be $1.50 m_{e}$ at all $k_{z}$, then $\gamma$ comes to $1.03 \mathrm{~mJ} / \mathrm{mol}^{-\mathrm{K}^{2}}$.

Resistive anisotropy. In the relaxation time approximation the expected anisotropy of a corrugated cylindrical $\mathrm{FS}$ is given by:

$$
\rho_{c} / \rho_{a b}=\left[d^{2} / 2 \sum_{\mu, \nu>0} k_{\mu \nu}^{2}\left(1+\delta_{\mu 0}\right)\right]^{-1},
$$

where $\delta_{\mu 0}$ is the Kronecker delta [20] and $d$ the interlayer spacing. Using the $k_{\mu \nu}$ from Table 1 of the main text yields $\rho_{c} / \rho_{a b}=241(13)$. The observed resistive anisotropy is larger (Fig. 5), especially below $25 \mathrm{~K}$, where it rises to more than 1000 . The suppression of Umklapp processes described in the main text may play a role in enhancing the anisotropy, and the larger anisotropy at low temperatures suggests that the defect scattering cross sections are themselves anisotropic. Overall, however, the scale of the resistive anisotropy of $\mathrm{PdCoO}_{2}$ should be the subject of further investigation.

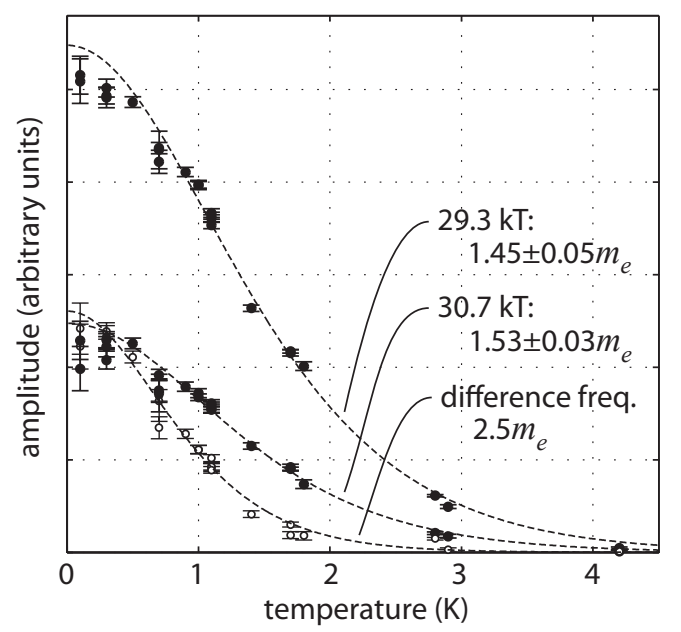

FIG. 4: Fits of dHvA amplitudes at $\theta=-6^{\circ}$, calculated over the field range 12.86-15 T, to the Lifshitz-Kosevich form, for the indicated frequencies. The masses resulting from the fits are indicated in the figure.

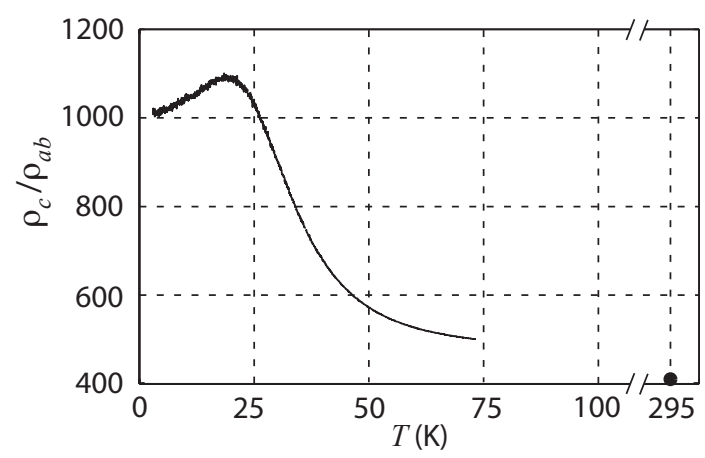

FIG. 5: Resistive anisotropy $\rho_{c} / \rho_{a b}$ against temperature. The error from sample geometrical factors is $10 \%$.

Tight-binding model. In the text of the paper we describe how strong interlayer next-nearest-neighbor $(n n n)$ coupling, as would result from Co-mediated hopping, would give a large $k_{31}$. For confirmation, we performed a tight-binding calculation. The transfer integrals included are in-plane $n n$ and $n n n$ coupling, $t_{n n} \equiv 1$ and $t_{n n n}$, and interlayer $n n$ and $n n n$ coupling, $t_{z}$ and $t_{z z} \cdot t_{n n n}=-0.23$ and $E_{F}=0.22$ approximately (although not uniquely) reproduce the half-filling and hexagonal shape of the Fermi surface. We calculate the FS for two sets of parameters, and obtain the harmonic components:

(1) $\left(t_{n n}, t_{n n n}, t_{z}, t_{z z}\right)=(1,-0.23,0.042,0.011)$ : $k_{60}=0.039, k_{01}=0.0106, k_{31}=0.0011 \AA^{-1}$.

(2) $\left(t_{n n}, t_{n n n}, t_{z}, t_{z z}\right)=(1,-0.23,0,0.04)$ : $k_{60}=0.039, k_{01}=0.0082, k_{31}=0.0103 \AA^{-1}$.

In the second set, where $t_{z z}$ dominates $t_{z}, k_{31}>k_{01}$, in disagreement with the experimental data.

Dingle analysis. In attempting a Dingle analysis, we concluded that the dHvA amplitudes are probably limited by phase smearing rather than impurity scattering: to observe a Dingle mean free path of $20 \mu \mathrm{m}$ would require a field homogeneity better than one in $10^{5}$. Such high homogeneity would be difficult to achieve even in the applied field, and $\mathrm{PdCoO}_{2}$ can contain Co-based impurity phases that are likely to increase the internal field inhomogeneity [9, 11]. Our dHvA amplitudes, after the effects of finite temperature and measurement time constant are removed, are shown in Fig. 6. Impurity scattering should yield a straightforward exponential decay, and fits to the data yield Dingle mean free paths of 5500 and $7000 \AA$ for the 29.3 and $30.7 \mathrm{kT}$ frequencies, respectively. However the exponential fits do not fit very well: the $29.3 \mathrm{kT}$ amplitude shows pronounced minima at $\approx 0.08$ and $0.11 \mathrm{~T}^{-1}$, and the $30.7 \mathrm{kT}$ amplitude appears to have minima at similar locations. This double-minimum form persists over a wide range of $\theta$, and so is not due to a small FS warping: it is the reason that many of 
the frequencies in Fig. 2 are double peaks with a $\sim 60 \mathrm{~T}$ splitting. Nor is it due to magnetic interaction: it persists to $T \sim 3 \mathrm{~K}$. Phase smearing is a possibility, with the minima being resonances where destructive interference is maximum, however the origin of this possible phase smearing is unclear.

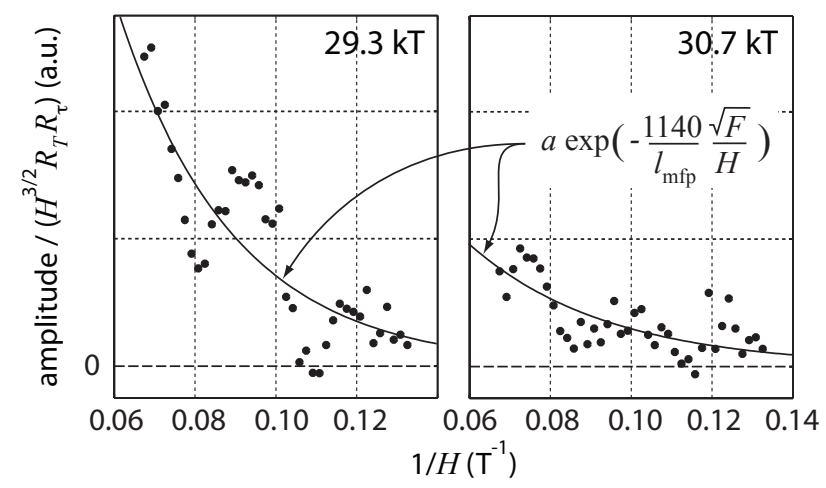

FIG. 6: Amplitudes of the 29.3 and $30.7 \mathrm{kT}$ peaks against $1 / H$, obtained by Fourier transforming over windows of width $0.00167 \mathrm{~T}^{-1} . R_{T}$ is the Lifshitz-Kosevich temperature attenuation form, with $T=0.7 \mathrm{~K}$, and $R_{\tau}$ is the attenuation due to the measurement time constant. The solid lines are fits to the standard Dingle attenuation; $F$ is the dHvA frequency in tesla, $l_{\mathrm{mfp}}$ the Dingle mean free path in $\AA$, and $H$ the field in tesla. 